\title{
GENERALIZATION OF A TRANSFORMATION FORMULA FOUND BY BAILLON AND BRUCK
}

\author{
ArJun K. Rathie and Yong Sup Kim
}

ABSTRACT. We aim mainly at presenting a generalization of a transformation formula found by Baillon and Bruck. The result is derived with the help of the well-known quadratic transformation formula due to Gauss.

\section{Introduction}

There was an open problem posed by Baillon and Bruck [1, Eq.(9.10)] who needed to verify the following hypergeometric identity

$$
\begin{aligned}
& { }_{2} F_{1}\left[\begin{array}{c}
\frac{1}{2}, \\
2
\end{array}{ }^{-m} ; 4 x(1-x)\right] \\
= & (m+1)(1-x) x^{2 m-1}{ }_{2} F_{1}\left[\begin{array}{c}
-m,-m \\
2
\end{array} ;\left(\frac{1-x}{x}\right)^{2}\right] \\
& +(2 x-1) x^{2 m-1}{ }_{2} F_{1}\left[\begin{array}{c}
-m,-m \\
1
\end{array}\left(\frac{1-x}{x}\right)^{2}\right]
\end{aligned}
$$

in order to derive a quantitative form of the Ishikawa-Edelstein-O'Brain asymptotic regularity theorem. Using Zeilberger's algorithm [4], Baillon and Bruck [1] gave a computer proof of this identity which is the key to the integral representation [1, Eq.(2.1)] of their main theorem. In 1995, Paule [2] gave the proof of (1.1) by using classical hypergeometric machinery by means of the following contiguous relations:

$$
\begin{gathered}
\frac{a b z}{c(c-1)}{ }_{2} F_{1}\left[\begin{array}{c}
a+1, b+1 \\
c+1
\end{array} ; z\right]={ }_{2} F_{1}\left[\begin{array}{c}
a, b \\
c-1
\end{array} ; z\right]-{ }_{2} F_{1}\left[\begin{array}{c}
a, b \\
c
\end{array} ; z\right], \\
{ }_{2} F_{1}\left[\begin{array}{c}
a+1, b \\
c+1
\end{array} ; z\right]=\frac{a-c}{a}{ }_{2} F_{1}\left[\begin{array}{c}
a, b \\
c+1
\end{array} ; z\right]+\frac{c}{a}{ }_{2} F_{1}\left[\begin{array}{c}
a, b \\
c
\end{array} ; z\right],
\end{gathered}
$$

Received April 18, 2008.

2000 Mathematics Subject Classification. Primary 33C20, 33C60; Secondary 33C70, $33 \mathrm{C} 65$.

Key words and phrases. Gauss's transformation formula, asymptotic regularity theorem, Zeilberger's algorithm, generalized hypergeometric functions.

This paper was supported by Wonkwang University in 2008. 
and the following well-known quadratic transformation formula [3] due to Gauss

$$
{ }_{2} F_{1}\left[\begin{array}{l}
a, b \\
2 b
\end{array} ; \frac{4 z}{(1+z)^{2}}\right]=(1+z)^{2 a}{ }_{2} F_{1}\left[\begin{array}{c}
a, a-b+\frac{1}{2} ; z^{2} \\
b+\frac{1}{2}
\end{array} .\right.
$$

The aim of this short paper is to provide a generalization of (1.1) by employing the transformation formula (1.4).

\section{Main result}

The following a generalization of the result (1.1) will be established:

$$
\begin{aligned}
& { }_{2} F_{1}\left[\begin{array}{cc}
a, b \\
2 b+1
\end{array} ; 4 z(1-z)\right] \\
= & z^{-2 a}\left\{{ }_{2} F_{1}\left[\begin{array}{c}
a, a-b+\frac{1}{2} ;\left(\frac{1-z}{z}\right)^{2} \\
b+\frac{1}{2}
\end{array}\right]\right. \\
& \left.+\frac{2 a(1-z)}{(2 b+1) z}{ }_{2} F_{1}\left[\begin{array}{c}
a+1, a-b+\frac{1}{2} \\
b+\frac{3}{2}
\end{array} ;\left(\frac{1-z}{z}\right)^{2}\right]\right\} .
\end{aligned}
$$

\section{Proof of (2.1)}

In order to prove the main result (2.1), we proceed as follows. From (1.2), we obtain the following relation:

$$
{ }_{2} F_{1}\left[\begin{array}{c}
a, b \\
2 b+1
\end{array} ; x\right]={ }_{2} F_{1}\left[\begin{array}{l}
a, b \\
2 b
\end{array} ; x\right]-\frac{a x}{2(2 b+1)}{ }_{2} F_{1}\left[\begin{array}{c}
a+1, b+1 \\
2 b+2
\end{array} ; x\right] .
$$

Now, put $x=-\frac{4 y}{(1-y)^{2}}$, we get

$$
\begin{gathered}
{ }_{2} F_{1}\left[\begin{array}{c}
a, b \\
2 b+1
\end{array} ;-\frac{4 y}{(1-y)^{2}}\right]={ }_{2} F_{1}\left[\begin{array}{c}
a, b \\
2 b
\end{array} ;-\frac{4 y}{(1-y)^{2}}\right] \\
+\frac{2 a y}{(2 b+1)(1-y)^{2}}{ }_{2} F_{1}\left[\begin{array}{c}
a+1, b+1 \\
2 b+2
\end{array} ;-\frac{4 y}{(1-y)^{2}}\right] .
\end{gathered}
$$

Multiplying both sides of $(3.2)$ by $(1-y)^{-2 a}$, we get

$$
\begin{aligned}
& (1-y)_{2}^{-2 a} F_{1}\left[\begin{array}{c}
a, b \\
2 b+1
\end{array} ;-\frac{4 y}{(1-y)^{2}}\right]=(1-y)^{-2 a}{ }_{2} F_{1}\left[\begin{array}{c}
a, b \\
2 b
\end{array} ;-\frac{4 y}{(1-y)^{2}}\right] \\
& +\frac{2 a y}{(2 b+1)}(1-y)^{-2(a+1)}{ }_{2} F_{1}\left[\begin{array}{c}
a+1, b+1 \\
2 b+2
\end{array} ;-\frac{4 y}{(1-y)^{2}}\right] .
\end{aligned}
$$

Now it is easy to see that the two ${ }_{2} F_{1}$ 's on the right hand side of (3.3) can be evaluated with the help of the Gauss' quadratic transformation formula (1.4), 
we get

$$
\begin{aligned}
& (1-y)^{-2 a}{ }_{2} F_{1}\left[\begin{array}{c}
a, b \\
2 b+1
\end{array} ;-\frac{4 y}{(1-y)^{2}}\right]={ }_{2} F_{1}\left[\begin{array}{c}
a, a-b+\frac{1}{2} \\
b+\frac{1}{2}
\end{array} y^{2}\right] \\
& +\frac{2 a y}{(2 b+1)}{ }_{2} F_{1}\left[\begin{array}{c}
a+1, a-b+\frac{1}{2} \\
b+\frac{3}{2}
\end{array} y^{2}\right]
\end{aligned}
$$

which can be written as

$$
\begin{aligned}
& { }_{2} F_{1}\left[\begin{array}{c}
a, b \\
2 b+1
\end{array} ;-\frac{4 y}{(1-y)^{2}}\right]=(1-y)^{2 a}\left\{{ }_{2} F_{1}\left[\begin{array}{c}
a, a-b+\frac{1}{2} \\
b+\frac{1}{2}
\end{array} y^{2}\right]\right. \\
& \left.\quad+\frac{2 a y}{(2 b+1)}{ }_{2} F_{1}\left[\begin{array}{c}
a+1, a-b+\frac{1}{2} ; y^{2} \\
b+\frac{3}{2}
\end{array}\right]\right\} .
\end{aligned}
$$

Now, changing $y$ to $-y$, we get

$$
\begin{aligned}
& { }_{2} F_{1}\left[\begin{array}{c}
a, b \\
2 b+1
\end{array} ; \frac{4 y}{(1+y)^{2}}\right]=(1+y)^{2 a}\left\{{ }_{2} F_{1}\left[\begin{array}{c}
a, a-b+\frac{1}{2} \\
b+\frac{1}{2}
\end{array} y^{2}\right]\right. \\
& -\frac{2 a y}{(2 b+1)}{ }_{2} F_{1}\left[\begin{array}{c}
a+1, a-b+\frac{1}{2} ; y^{2} \\
b+\frac{3}{2}
\end{array}\right\} \text {. }
\end{aligned}
$$

Finally, taking $y=\frac{1-z}{z}$ and we, after a little simplification, have

$$
\begin{aligned}
& { }_{2} F_{1}\left[\begin{array}{c}
a, b \\
2 b+1
\end{array} ; 4 z(1-z)\right]=z^{-2 a}\left\{{ }_{2} F_{1}\left[\begin{array}{c}
a, a-b+\frac{1}{2} \\
b+\frac{1}{2}
\end{array} ;\left(\frac{1-z}{z}\right)^{2}\right]\right. \\
& \left.\quad-\frac{2 a(1-z)}{(2 b+1) z}{ }_{2} F_{1}\left[\begin{array}{c}
a+1, a-b+\frac{1}{2} ;\left(\frac{1-z}{z}\right)^{2} \\
b+\frac{3}{2}
\end{array}\right]\right\} .
\end{aligned}
$$

This completes the proof of (2.1).

\section{Special case}

In our main transformation formula (2.1), if we take $a=-m$ and $b=\frac{1}{2}$, we obtain

$$
\begin{aligned}
& { }_{2} F_{1}\left[\begin{array}{c}
\frac{1}{2},-m \\
2
\end{array} ; 4(1-z)\right]=z^{2 m}\left\{{ }_{2} F_{1}\left[\begin{array}{c}
-m,-m \\
1
\end{array}\right]\left(\frac{1-z}{z}\right)^{2}\right] \\
& \left.+m\left(\frac{1-z}{z}\right){ }_{2} F_{1}\left[\begin{array}{c}
-m,-m+1 \\
2
\end{array} ;\left(\frac{1-z}{z}\right)^{2}\right]\right\} .
\end{aligned}
$$

Equation (4.1) is an alternate form of the result (1.1) due to Baillon and Bruck.

Remark. The result (1.1) in its exact form can be obtained from (4.1) by using (1.3) with $a=b=-m$ and $c=1$. 


\section{References}

[1] J.-B. Baillon and R. E. Bruck, The rate of asymptotic regularity is o $\left(\frac{1}{\sqrt{n}}\right)$, Theory and Applications of Nonlinear Operators of Accretive and Monotone Type, Lecture Notes in Pure and Appl. Math., Dekker, New York, 178 (1996), 51-81.

[2] P. Paule, A classical hypergeometric proof of an important transformation formula found by Baillon and Bruck, Theory and Applications of Nonlinear Operators of Accretive and Monotone Type, Lecture Notes in Pure and Appl. Math., Dekker, New York, 178 (1996), $241-242$.

[3] E. D. Rainville, Special Functions, The Macmillan Company, New York, 1960.

[4] D. Zeilberger, A fast algorithm for proving terminating hypergeometric identities, Discr. Math. 80 (1990), 207-211.

ArJun K. Rathie

Department of Mathematics

Vedant College of Engineering and Technology Tulsi-323021, Dist. Bundi, Rajasthan State, India

E-mail address: akrathie@rediffmail.com

Yong Sup KIM

Department of Mathematics Education

WONKWANG UNIVERSITY

IKSAN 570-749, KOREA

E-mail address: yspkim@wonkwang.ac.kr 